\title{
Le radar comme aide à l'estimation quantitative des précipitations. Exemple du radar numérisé de Dammartin-en-Goële
}

\section{The radar as an aid to quantitative estimation of precipitation. The example of the Dammartin-en-Goelle digital radar}

\author{
G. Floch \\ Station radar météorologique, Dammartin-en-Goële
}

Le Nord de la France est soumis à un climat propice à des inondations hivemales du fait de la répétition des précipitations quasi-journalières pendant de longues périodes, quand elles ne sont pas encore accentuées par la fonte de neige. Il arrive également que de fortes pluies de courte durée produisent des crues locales. Dans les deux cas, les réseaux de mesures pluviométriques sont insuffisants pour bien appréhender la lame d'eau tombée et la gérer au mieux; c'est ce que l'on souhaite améliorer surtout en milieu urbain. Un radar de précipitations devrait être en mesure d'apporter un complément intéressant sur un domaine aussi important. Le passage à la pratique montre qu'un certain nombre d'écueils se présentent quand on tente d'utiliser un équipement dont la vocation première consiste à surveiller les évolutions des phénomènes précipitants pour ses utilisations météorologiques.

\section{Les moyens}

Un radar de $10 \mathrm{~cm}$ de longueur d'onde MELODI, installé à la Station Météorologique de Dammartin-enGoële, est doté d'un équipement SAPHYR de numérisation et de traitements des données en temps réel. Un moniteur de télévision couleur visualise les informations qui sont également transmises par lignes téléphoniques et enregistrées sur bandes magnétiques, à des cadences différentes selon les utilisations prévues; par exemple une image toutes les 5 minutes pour des traitements hydrologiques.

Six techniciens météorologistes se relayent 24 heures sur 24 pour suivre l'évolution de la situation pluviométrique et en informer qualitativement les utilisateurs: par téléphone, télex, répondeur téléphonique ou transmission d'images commentées. Le passage à la prévision quantitative est très souhaité.
Un deuxième microcalculateur permet aux moments disponibles, de visionner les situations et de leur appliquer des traitements climatologiques ou hydrologiques. Les contraintes d'exploitation météorologique ne permettent de progresser que très lentement.

\section{Les enregistrements}

Jusqu'en fin 1982, l'archivage se faisait en azimut/ distance. Un tour d'antenne était enregistré toutes les 5 minutes et se composait comme suit :

- 256 portes de $500 \mathrm{~m}$ par intégration de 64 tirs consécutifs en 256 niveaux,

- vitesse de rotation voisine de 2 tours par minute (commande manuelle).

Les études dont il est fait état dans le présent rapport ont été menées avec ce type d'enregistrement.

Récemment, l'équipement a été modifié et réalise désormais deux modes d'archivage. L'image télévision numérique $(256 \times 256)$ en 16 niveaux est mémorisée sur bandes toutes les 15 minutes en vue de visionner rapidement un épisode pluvieux. Une deuxième application consiste à "cumuler" les images en vue de déterminer les zones arrosées. Le document obtenu constitue une aide pour le climatologiste chargé du contrôle des relevés pluviométriques quotidiens. Le deuxième mode d'archivage est conçu à des fins hydrologiques. Un tour d'antenne est saisi toutes les 5 minutes et possède les caractéristiques suivantes:

- 1024 portes de $250 \mathrm{~m}$ par intégration de 16 tirs consécutifs en 256 niveaux,

- vitesse de rotation de 3 tours par minute (commande au tomatique).

Cette densité d'archivage devrait permettre de déter- 
miner le mode de fonctionnement optimal au stade opérationnel de l'utilisation hyd rologique.

\section{Première tentative d'estimation pluviométrique}

Cette étude cherchait à faire correspondre à chaque niveau de réflectivité une quantité de pluie tombée en 5 minutes de manière à obtenir des valeurs voisines de celles relevées aux pluviomètres en 24 heures. On a fixé dix seuils $i$ de réflectivité auxquels correspondent les intensités $x_{i}$ de pluie, puis calculé le temps total $t_{i}$ passé à chacun des niveaux. La quantité totale de pluie en un point donné serait égale à :

$$
P=x_{1} t_{1}+x_{2} t_{2}+\cdots+x_{i} t_{i}+\cdots+x_{10} t_{10}
$$

En effectuant ce calcul en 10 points on obtient un système de 10 équations. Ces points étant choisis en des lieux où on a connaissance de la valeur de pluie, cela permet de résoudre le système d'équations en donnant à $P$ les valeurs observées. Les résultats matérialisés sur un graphique ont permis de tracer une courbe de correspondance entre les réflectivités et les intensités de pluie obtenue par échantillonnage de 5 minutes.

On a ensuite appliqué cette correspondance en des points de grille d'une zone. La critique des résultats a rapidement mis en évidence un certain nombre de problèmes à résoudre.

\section{Problèmes apparus en traitements hydrologiques}

\section{Les échos provoqués par le sol:}

Les premiers kilomètres autour d'un radar sont inutilisables en hydrologie du fait de la réflexion des ondes sur les objets atteints par le faisceau. Dans le cas présent, le rayon de cette zone parasitée est supérieur à $50 \mathrm{~km}$. Mais au-delà de cette partie centrale, certaines constructions particulièrement élevées (pylones) produisent encore des échos isolés qui faussent la mesure. Enfin, lors des propagations anormales, une partie du faisceau est réfléchie vers le sol et produit des échos à des distances bien plus grandes. Ceci devient particulièrement génant lorsqu'une précipitation co incide avec l'endroit où un tel phénomène se produit.

\section{Les masques :}

Le lieu d'implantation d'un radar météorologique résultant d'un compromis entre la qualité du site et certains impératifs, il n'est pas rare de trouver des objets ou collines proches qui rendent le radar aveugle, au moins dans la partie inférieure du faisceau. L'utilisation quand elle est possible qualitativement, parait ici fort compromise pour l'hydrologie; pour le moins, la correspondance obtenue dans les autres directions devra y être modulée.

\section{Les calages en azimut et distance :}

Il est indispensable que ces deux paramètres soient parfaitement recopiés par les codeurs et que le radar soit lui-même bien calé. Les différences que nous avons décelées atteignaient 2 degrés.

\section{La correction en distance :}

Quand différentes hypothèses et approximations sont vérifiées, l'énergie réfléchie est inversement proportionnelle au carré de la distance. Pour s'en affranchir, une correction parfaite doit être appliquée.

\section{Dimension du faisceau :}

Il n'est pas nécessaire que le faisceau soit complètement intercepté par une précipitation pour produire un écho. Ceci a pour effet d'agrandir la zone de pluie d'une grandeur pouvant atteindre la moitié du faisceau de part et d'autre. Il est donc probable que dans bien des cas, un point en bordure de la pluie se verra pourtant affecté d'une réflectivité. $\mathrm{Ce}$ phénomène prendra de limportance avec la distance mais il est possible qu'il soit compensé par de faibles pluies de bordure qui ne seraient pas détectées.

\section{Décalage du au vent :}

Sans l'avoir analysé, il n'est pas exclu que le vent ait un effet non négligeable sur la position d'une zone de pluie par rapport à la région effectivement arrosée.

\section{Etalonnage du radar :}

Pour extrapoler d'une situation à une autre, il est impératif que l'étalonnage de la chaîne de réception du radar soit d'une grande stabilité et donc vérifiée régulièrement. Sinon, il serait nécessaire de recalibrer le radar en permanence.

\section{Calage de l'angle de site :}

Dans le même ordre d'idée, l'angle d'émission dans le plan vertical devra être le plus adapté à l'hydrologie et conservé par l'équipement d'une façon très précise.

\section{Echos temporaires autres que de précipitations :}

Des avions, des oiseaux, de la fumée, ... . produisent des échos qui, répétés (approche d'aérodrome), apportent des erreurs importantes dans les estimations.

\section{Echos n'atteignant pas le sol:}

Certaines situations météorologiques sont génératrices de pluies qui n'atteignent pas le sol et qui pourront être décelées à certaines distances où l'altitude du faisceau co incide avec la présence de ces pluies.

\section{Précipitations solides :}

Nos études écartent pour l'instant toutes les situations qui ont apporté des précipitations solides car elles compliquent encore la résolution du problème. 


\section{Sélection de la valeur à affecter en un point donné :}

Les enregistrements étant commandés par une horloge, les azimuts correspondant aux résultats d'intégration varient d'un tour d'antenne au suivant. Pour obtenir la suite des réflectivités à affecter en un point donné, on doit sélectionner un azimut proche de celui du point. Pour disposer d'une valeur toutes les 5 minutes, on choisira entre les deux azimuts successifs entourant le point, celui qui est le plus proche. L'ouverture de l'angle incluant les azimuts affectés pour un lieu sera égal à l'angle parcouru par le radar pendant le temps d'intégration soit $256 \mathrm{~ms}$ correspondant, pour une vitesse de rotation de 2 tours par minute, à environ 3 degrés. Ceci a pour effet d'augmenter encore l'incertitude sur la coincidence entre écho et précipitation.

\section{Echantillonnage dans le temps :}

Pour permettre simultanément l'exploitation météorologique et l'enregistrement hydrologique, il n'est pas possible d'enregistrer plus d'un tour toutes les 5 minutes. Ceci ne constitue sans doute pas un échantillonnage suffisant, en particulier s'il s'agit d'une averse de forte intensité, très évolutive et de faibles dimensions horizontales. Il n'est donc pas étonnant dans une situation instable d'obtenir des estimations particulièrement décevantes.

\section{Analyse de la qualité de détection du radar}

Cette étude a été menée sur le département de l'Eure et Loir (pas de masque ni d'échos permanents). On a tenu compte des décalages d'angle et de distance découverts sur des échos de sol dans ces directions. Pour vérifier ces corrections sur des pluies, nous avons recherché les heures de début et de fin d'échos en un lieu donné et les avons comparé à celles observées sur le site. Pour les Stations Météorologiques de Chartres et Chateaudun, la journée du 5 mai 1982, était intéressante car il s'agissait d'une situation à averses nombreuses et de petites dimensions. A partir d'une grille autour de ces Stations nous avons recherché le point qui donnait la meilleure coincidence entre les passages pluvieux et les échos. Etant donné la dimension du faisceau plusieurs points ont donné des résultats très peu différents, mais leur concentration autour de la Station confirme bien les décalages. On s'aperçoit aussi qu'à $150 \mathrm{~km}$ des pluies faibles sont encore décelées, ce qui peut laisser espérer des estimations hydrologiques jusqu'à de telles distances, (voir en annexe le tableau comparatif des pluies et des échos à Chartres et Chateaudun pour le 5/5/82). Toutes les pluies sont décelées et on trouve des échos sans pluies qui semblent correspondre au phénomène de bordure signalé plus haut.

\section{Projet}

Le projet final prévoit l'estimation de la pluviométrie sur les régions non perturbées par des échos permanents ou des secteurs aveugles. Ce but, sans être déraisonnable, parait néanmoins bien lointain en raison de toutes les embûches rencontrées et du peu de temps qu'il est possible d'y consacrer.

Annexe

Tableau comparatif des heures de début et de fin de pluies à Chartres et Chateaudun et des heures de détection par le radar de Dammartin-en-Goële le 5 mai 1982

\begin{tabular}{|c|c|c|c|c|c|c|}
\hline \multicolumn{3}{|c|}{$\begin{array}{c}\text { Chartres } \\
232,2^{\circ} / 108 \mathrm{~km}\end{array}$} & \multicolumn{4}{|c|}{$\begin{array}{c}\text { Chateaudun } \\
220,9^{\circ} / 144 \mathrm{~km}\end{array}$} \\
\hline $\begin{array}{l}\text { Début-fin } \\
\text { des pluies }\end{array}$ & $\begin{array}{l}\text { Hauteur } \\
\text { en } \mathrm{mm}\end{array}$ & $\begin{array}{l}\text { Présence } \\
\text { d'échos }\end{array}$ & $\begin{array}{l}\text { Début-fin } \\
\text { des pluies }\end{array}$ & $\begin{array}{l}\text { Hauteur } \\
\text { en mm }\end{array}$ & $\begin{array}{l}\text { Présence } \\
\text { d'échos }\end{array}$ & $\begin{array}{l}\text { Echos au point optimal } \\
220,7^{\circ} 144 \mathrm{~km}\end{array}$ \\
\hline & & $08-H 52$ & $08 \mathrm{H} 35-08 \mathrm{H} 40$ & 0,2 & & $08 \mathrm{H} 42$ \\
\hline $09 \mathrm{H} 20-09 \mathrm{H} 30$ & 0,4 & $09 H 22-09 H 32$ & $12 \mathrm{H} 5 \mathrm{O}-13 \mathrm{H} 10$ & 0,6 & $12 \mathrm{H} 57-13 \mathrm{HO} 2$ & $12 \mathrm{H} 57-13 \mathrm{HO} 2$ \\
\hline O9H55-10H05 & 0,0 & $\begin{array}{l}10 \mathrm{H} 07 \\
10 \mathrm{H} 32\end{array}$ & $\begin{array}{l}13 \mathrm{H} 35-13 \mathrm{H} 45 \\
15 \mathrm{H} 50-16 \mathrm{H} 00\end{array}$ & $\begin{array}{l}0,2 \\
1,4\end{array}$ & $\begin{array}{l}13 \mathrm{H} 32-13 \mathrm{H} 37 \\
15 \mathrm{H} 52\end{array}$ & $\begin{array}{l}13 \mathrm{H} 37 \\
15 \mathrm{H} 52-15 \mathrm{H} 57\end{array}$ \\
\hline $11 \mathrm{H} 05-11 \mathrm{H} 10$ & 1,0 & $10 \mathrm{H} 57-11 \mathrm{H} 12$ & $19 \mathrm{H} 10-19 \mathrm{H} 50$ & 3,4 & $19 \mathrm{H}_{12}-2 \mathrm{OHO} 2$ & $19 Y 17-20 \mathrm{HO} 2$ \\
\hline $13 \mathrm{H} 40.13 \mathrm{H} 45$ & 0,2 & $13 \mathrm{H} 37-13 \mathrm{H} 52$ & $20 H 50-21 \mathrm{H} 10$ & 0,4 & $21 \mathrm{HO} 2$ & $20 \mathrm{H} 57-21 \mathrm{HO}$ \\
\hline $14 \mathrm{HOO}-14 \mathrm{HOS}$ & 0,6 & $\begin{array}{l}14 \mathrm{HO} 7 \\
14 \mathrm{H} 32\end{array}$ & & & & \\
\hline $16 \mathrm{H} 15-16 \mathrm{H} 45$ & 2.8 & $\begin{array}{l}16 \mathrm{H} 12-16 \mathrm{H} 47 \\
20 \mathrm{H} 12-2 \mathrm{H} 17\end{array}$ & & & & \\
\hline
\end{tabular}

Nota : Les échos sont signalés par les heures de leurs premières et de leurs dernières présences dans les enregistrements. Une seule heure signifie que l'écho a été décelé et enregistré lors d'un tour d'antenne, laverse ayant pu néanmoins durer jusqu'à dix minutes. 


\section{Discussion}

Président : J. ETIENNE

M. FLOCH précise que, comme pour l'expérience de la Martinique, celle de Dammartin a été réalisée avec des radars exploités normalement pour d'autres buts, mais qui ont été utilisés pour essayer d'en tirer le maximum de possibilités, puis $M$. BISSONNIER évoque les questions de calibration et d'étalonnage. Il constate que la météorologie a tendance à vouloir faire des calibrations avec des pluviomètres, tandis qu'il lui semble que les hydrologues pourraient apporter quelque chose, dans la mesure où on pourrait choisir comme système de référence la mesure des débits; ceci serait particulièrement intéressant dans les cas de situations convectives.

Le Président ajoute que l'on pourrait faire des critiques dans le même style que $\mathrm{M}$. FLOCH sur la précision des pluviomètres pour les différents types de pluies.

M. DUJARDIN estime que la notion de zone arrosée, présentée dans les vues projetées en fin đ'exposé, lui paraît très intéressante. Ainsi, dans les pays ayant un faible réseau d'équipements au sol, et où les pluies sont assez localisées, il arrive que les hydrologues voient passer une crue mais ne sont pas capables de connaître le volume de pluie qui l'a occasionnée, car les plu- viomètres sont trop espacés. Grâce aux radars on pourrait cependant déterminer la zone d'influence de la perturbation qui s'est produite.

M. BISSONNIER évoque encore le cas des erreurs possibles dues au fait que certaines pluies n'atteignent pas le sol.

M. BEDIOT appelle l'attention sur le fait que l'imagerie radar apporte une vue d'ensemble du phénomène pluvieux qui fait réviser nos conceptions de critique des données de précipitations quotidiennes. Elle met en évidence les pratiques erronées qui consistaient, par exemple, à aligner les précipitations quotidiennes d'un département sur une même journée ou à réduire les valeurs ponctuelles extrêmes relevées par les observateurs, sous prétexte que ces valeurs ne paraissaient pas conformes à l'idée qu'on se faisait de la répartition spatiale de la pluie. Il s'agit là d'un piont très important dans la valorisation des données pluviométriques qui remontent à plus de cent ans : cela incite le "Bureau de l'eau" de la Météorologie, qui a la charge de cette question, à beaucoup de prudence dans la mise en oeuvre des méthodes de critique des données pluviométriques. 